\title{
Plasmonics-Enhanced UV Photocatalytic Water Purification
}

Ryan Brisbin ${ }^{1,3}$, Jenny Zhou ${ }^{1}$, Tiziana Bond ${ }^{1}$, Lars Voss ${ }^{1}$, Aaron J. Simon ${ }^{2}$, Ryan Baxter ${ }^{3}$, and Allan S.P. Chang ${ }^{*}, 1$

${ }^{1}$ Materials Engineering Division, Lawrence Livermore National Lab, Livermore, CA 94550

${ }^{2}$ E-Program, Global Security Directorate, Lawrence Livermore National Lab, Livermore, CA 94550

${ }^{3}$ Department of Chemistry and Chemical Biology, School of Natural Sciences, University of California, Merced, CA 95344, USA

S1.................. Kinetics methodology

S2-S3.............. Equations used in kinetic mapping

S4................. Manufacturer spectrum of UV lamp

S5-S6.............. SEM images of 120nm Al feature sizes before and after $10 \mathrm{~min}$. UV exposure in Methyl Orange solution

S7 ................. SEM images of $90 \mathrm{~nm} \mathrm{Al} \mathrm{feature} \mathrm{sizes} \mathrm{before} \mathrm{and} \mathrm{after} 10 \mathrm{~min}$. UV exposure in Methyl Orange solution

S8.................. Table of average feature sizes and gap sizes before and after UV exposure

S9.................. UV-Vis Absorption spectrum of Methyl Orange 


\section{Protocol for Determining Rate Enhancement in Methyl Orange Decomposition Experiments.}

\section{Kinetics Experiments}

All kinetics experiments were performed as a series of independent reactions with various exposure times. All experimental reactions consisted of a $15 \mathrm{~mL}$ aliquot from a fresh made bulk solution consisting of a 1:15 dilution of stock methyl orange (MO) solution with a concentration of $1 \% \mathrm{~W}: \mathrm{V}$. Kinetics experiments begin by placing the desired substrate ( blank $\mathrm{TiO}_{2}$, or $\mathrm{Al}$ patterned) into the bottom of a 6" borosilicate petri dish (catalytic side face up). Following substrate placement, a $15 \mathrm{~mL}$ aliquot of the diluted bulk solution was measured using a $15 \mathrm{~mL}$ volumetric flask and carefully poured into the petri dish. The addition of dilute MO solution must be done slowly and with care as rapid addition of solution cause substrate to lift off from the surface of the petri dish and float on solution.

Once addition of MO solution was accomplished and substrate remained submerged, petri dish was capped with the borosilicate top and covered in tinfoil. The reaction was allowed to equilibrate under the tinfoil covering for 10 minutes and transferred to the IntelliRay-600 UV shutter flood light chamber set to $35 \%$ power with no temperature input for UV exposure. Exposure time was set in seconds (Table S1) on the display. The door to the UV chamber was shut to allow UV exposure. Following the UV exposure reaction vessel was left in the UV chamber for an additional 2-3 minutes prior to removal. Following removal of the reaction from the UV chamber, the cap was removed, and a micro-pipette was used to transfer all liquid from reaction vessel to a clean $15 \mathrm{~mL}$ volumetric flask. Evaporative loss was assessed by volume loss and compensated for by addition of proportional milli-Q deionized water (DI).

Post volumetric normalization the flask was capped and inverted four time (4x) to ensure the homogeneity of the reaction solution.

One hundred (100) microliters were transferred into a cuvette followed by 900 microliters of DI water (1:10 dilution of post UV exposed solution) for use in a Perkin Elmer $950 \mathrm{UV}-\mathrm{V}$ is spectrophotometer with attached $60 \mathrm{~mm}$ integrating sphere for calibrated concentration assessment. Four (4) samples were taken from each reaction (time point) for establishment of standard deviation.

\section{Table S1}

\begin{tabular}{|l|}
\hline Minutes \\
\hline 0 \\
\hline 1 \\
\hline 3 \\
\hline 5 \\
\hline 7 \\
\hline 14 \\
\hline
\end{tabular}




\section{$\underline{\text { Equations for Mapping Kinetic Data }}$}

Following collection of the UV-Vis data, analysis was conducted via calculating absorption [A] from percent transmission. Absorption values at $\mathbf{4 6 9} \mathbf{n m}$ was compared to an externally generated calibration curve to ascertain methyl orange concentration. Equations were mapped to the concentration vs. time as shown in Figure S1A, equations for polynomial fitting are displayed in Table 1. Following curve fitting of the concentration/time plots, the slope was found by calculating the first derivative and using the slope as a proxy for decomposition rate k, which can be found in Figure S1B and Table 2.
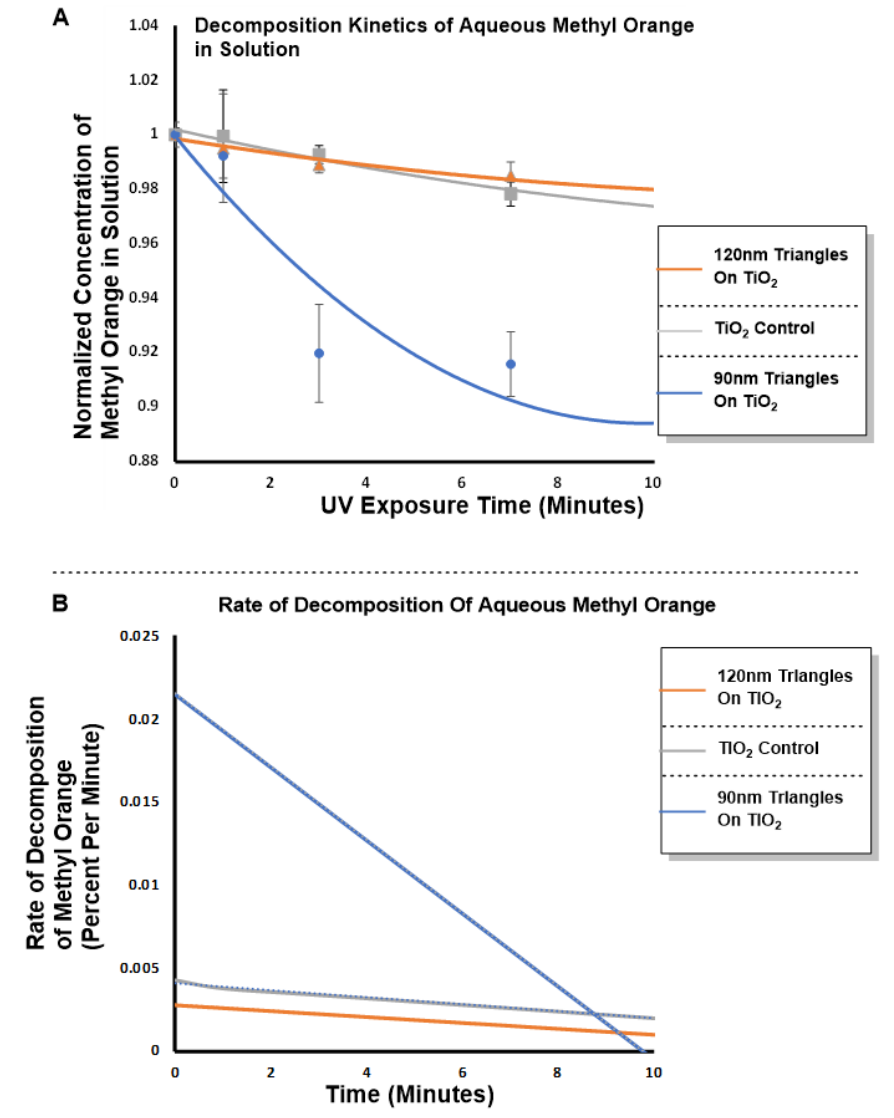

Figure S1

Table 1

\begin{tabular}{|c|c|}
\hline Experiment & Graphics Analysis \\
\hline $\mathrm{TiO}_{2}$ & $\mathrm{y}=0.0001 \mathrm{x}^{2}-0.004 \mathrm{x}+1.0018$ \\
\hline $\mathrm{TiO}_{2}$ Off Tuned & $\mathrm{y}=9 \mathrm{E}-05 \mathrm{x}^{2}-0.0028 \mathrm{x}+0.9985$ \\
\hline $\mathrm{TiO}_{2}$ Tuned & $\mathrm{y}=0.0011 \mathrm{x}^{2}-0.0215 \mathrm{x}+0.9994$ \\
\hline
\end{tabular}


Table 2

$\left.\begin{array}{|c|c|}\hline \text { Experiment } & \begin{array}{c}\text { Maximum Rate of } \\ \text { Decomposition (percent } \\ \text { decomposition minute }\end{array} \\ \hline \mathrm{TiO}_{2} \text { ) }\end{array}\right)$


Spectrum of UV-Lamp from Manufacturer

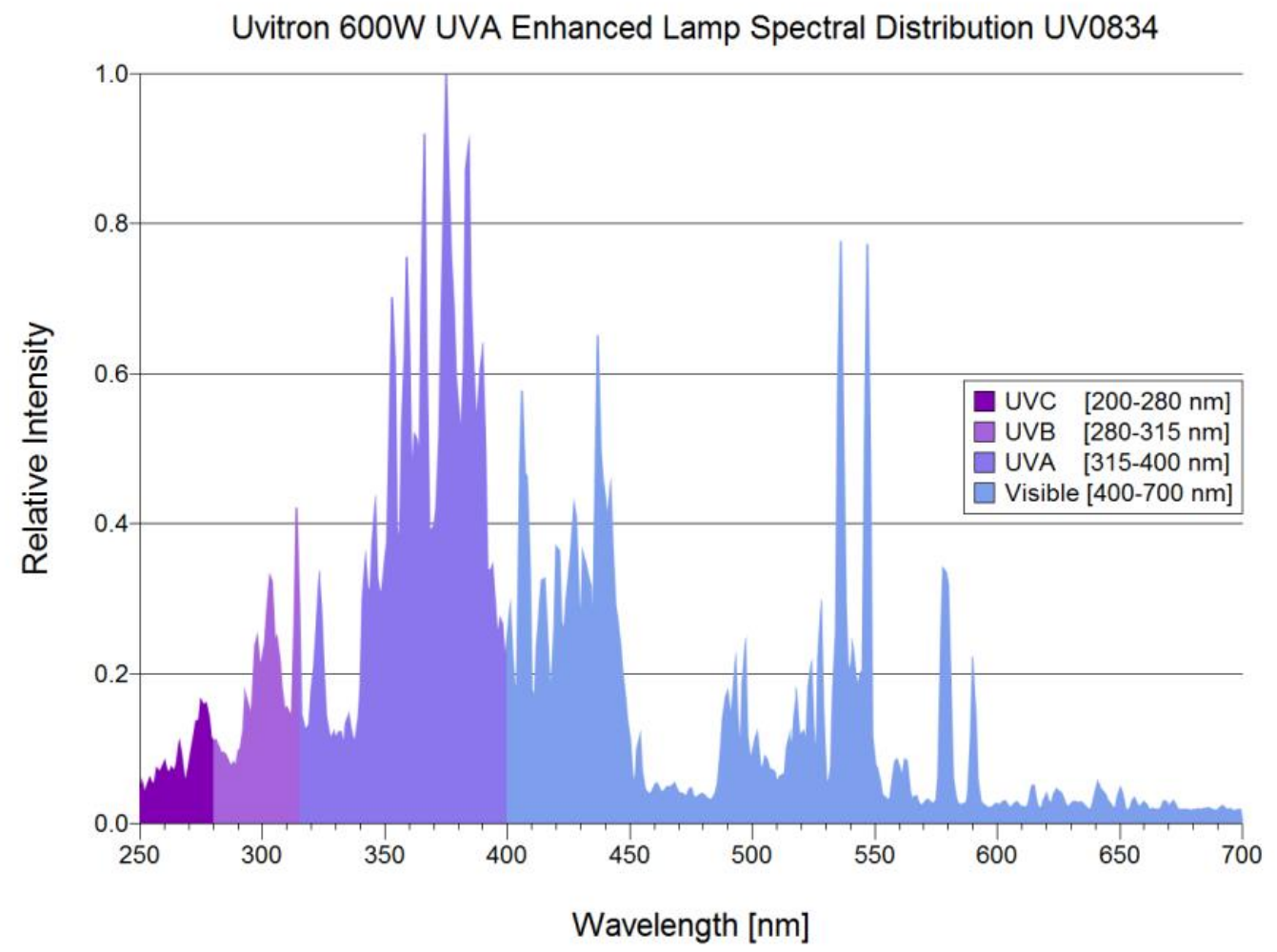




\section{SEM images of 120nm Al feature sizes before and after $10 \mathrm{~min}$. UV exposure in Methyl Orange} solution

\section{Methodology}

Patterned substrates were imaged with SEM before and after UV exposure to determine the average feature size and gap size. For each measurement, the feature and gap sizes on three identical chips were imaged and measured on two different locations on the chip. While some defects are present on the samples, only the triangular features were accounted for when collecting the measurements across the different samples. The two areas measured on each sample varied from one to another. However, the locations pertained to clusters of defect-free features where most of the activity will occur. Ultimately, the resulting measurements show a clear dimensional distinction between the two feature sizes before and after UV exposure.

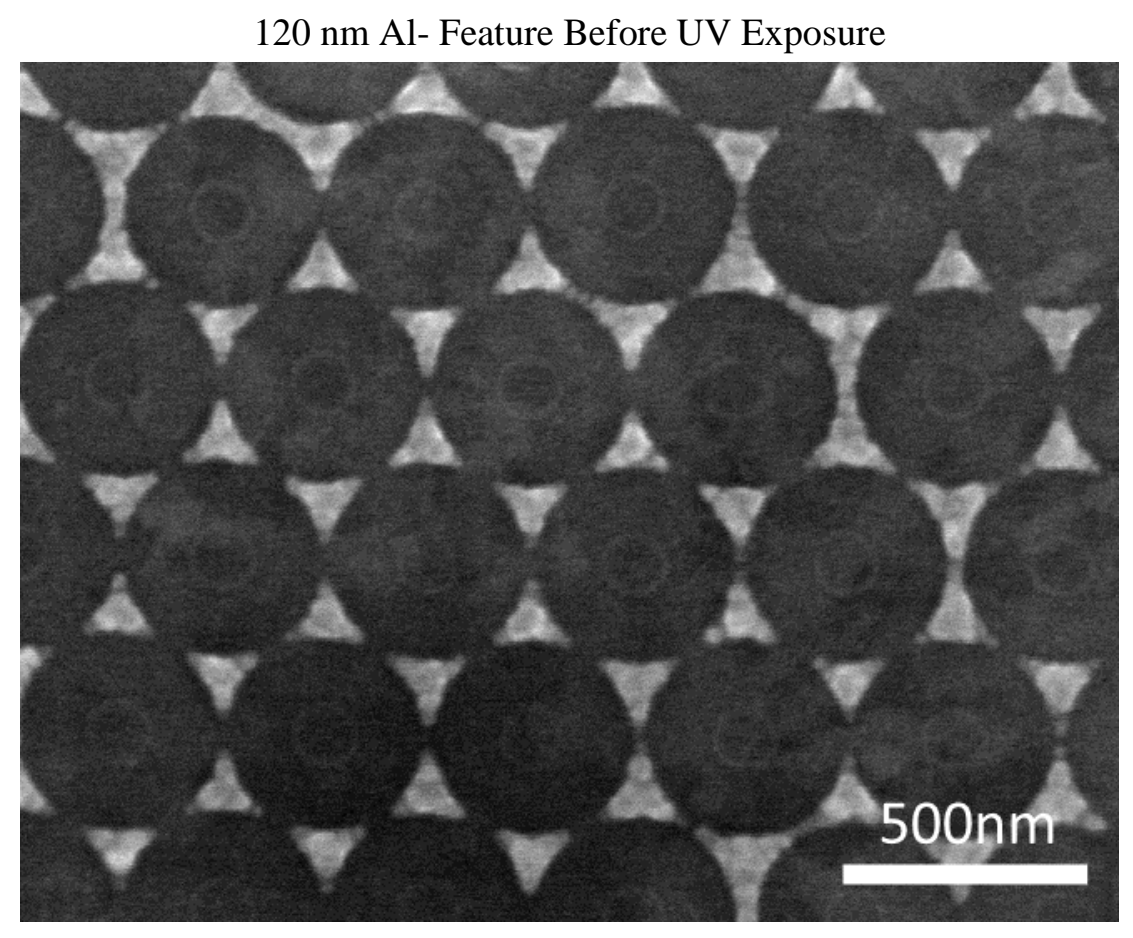


$120 \mathrm{~nm} \mathrm{Al-} \mathrm{Feature} \mathrm{After} \mathrm{UV} \mathrm{Exposure}$

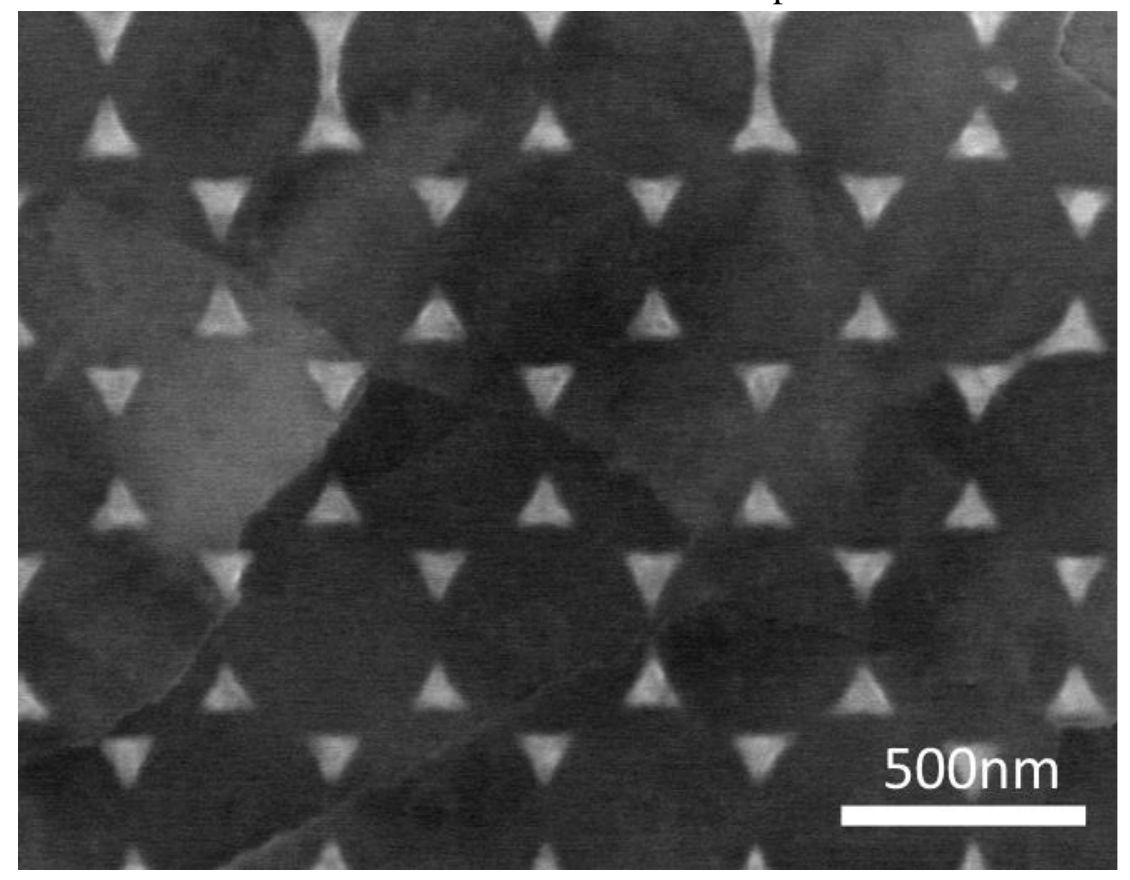


SEM images of 90nm Al feature sizes before and after $10 \mathrm{~min}$. UV exposure in Methyl Orange solution

90nm Al Feature Before UV Exposure

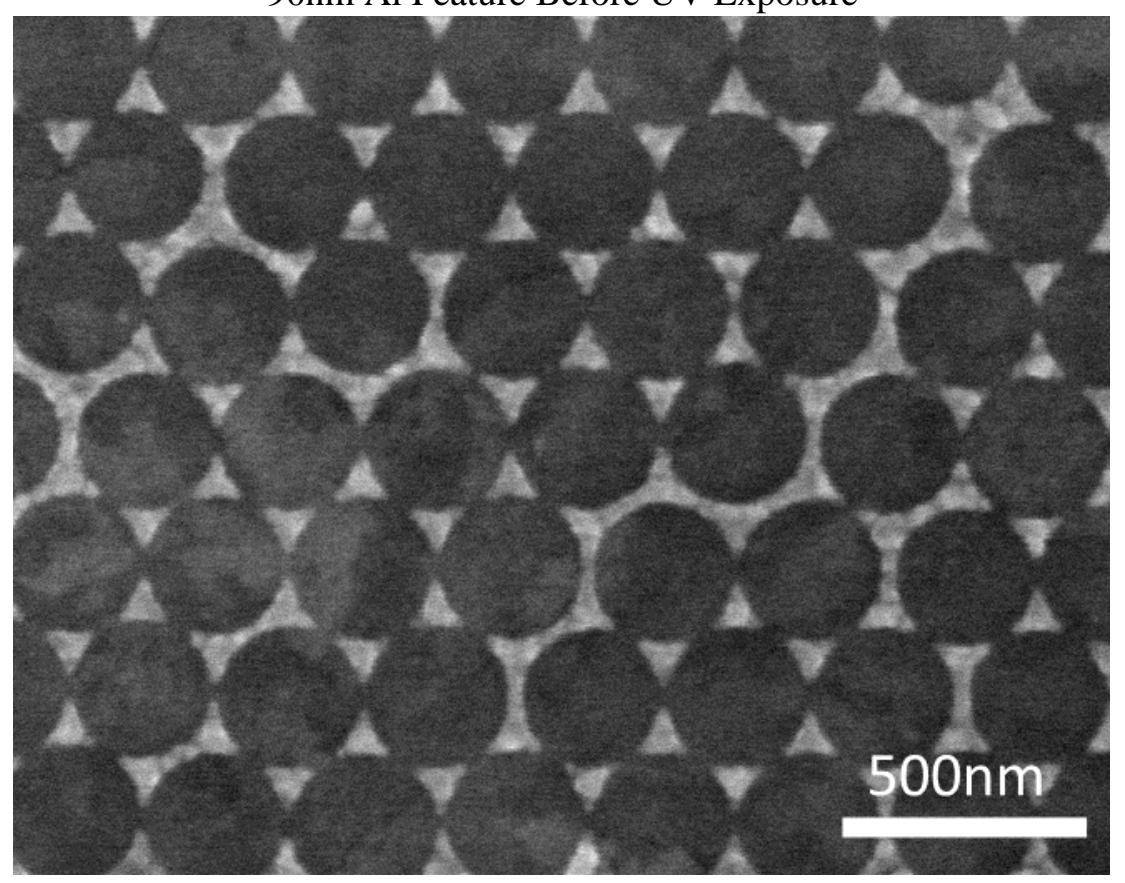

90nm Al Features After UV Exposure

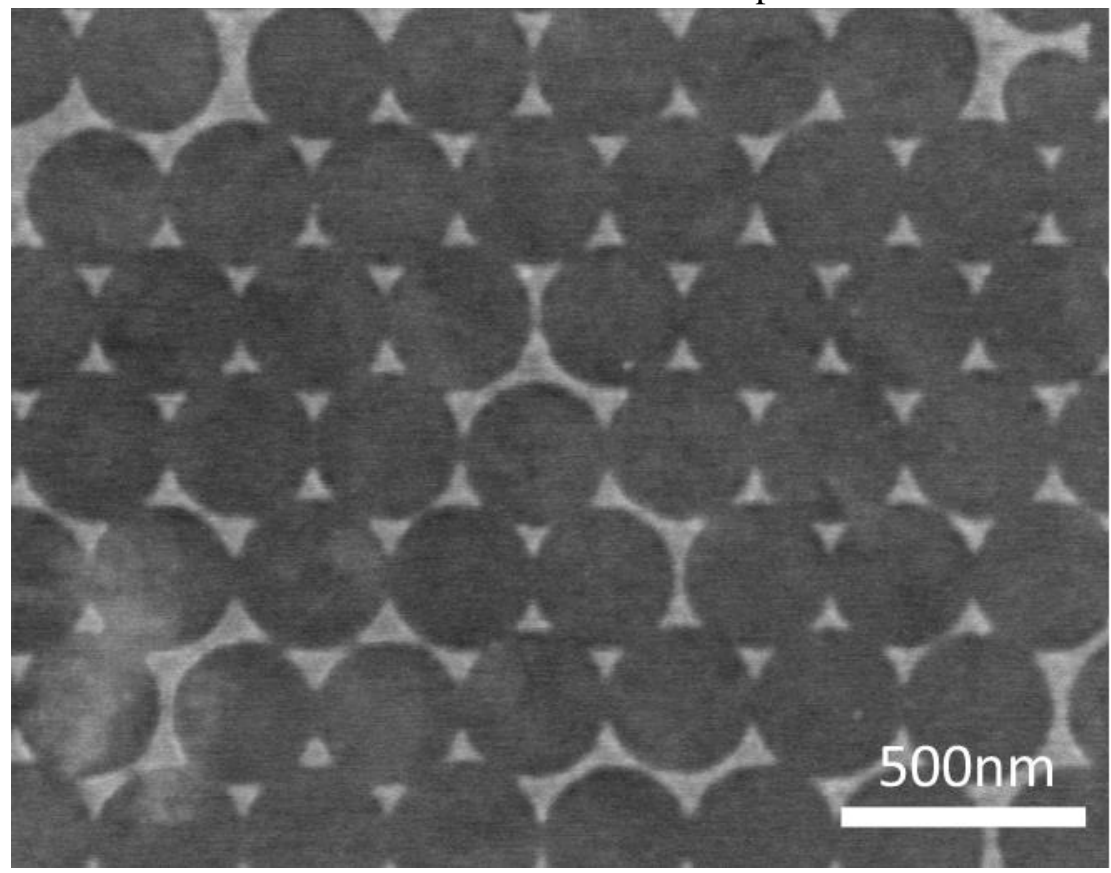




\begin{tabular}{|c|c|c|c|c|}
\hline \multirow{2}{*}{$\begin{array}{c}\text { Average } \\
\text { Feature Size } \\
\text { (nm) }\end{array}$} & \multicolumn{2}{|c|}{ *Feature Size (nm) } & \multicolumn{2}{c|}{$* *$ Gap (nm) } \\
\cline { 2 - 5 } & Pre-UV & Post-UV & Pre-UV & Post-UV \\
\hline 120 & $120.3 \pm 7.0$ & $94.2 \pm 6.7$ & $60.2 \pm 6.0$ & $101.7 \pm 12.0$ \\
\hline 90 & $90.4 \pm 5.0$ & $63.3 \pm 5.1$ & $39.8 \pm 4.1$ & $73.0 \pm 7.1$ \\
\hline
\end{tabular}

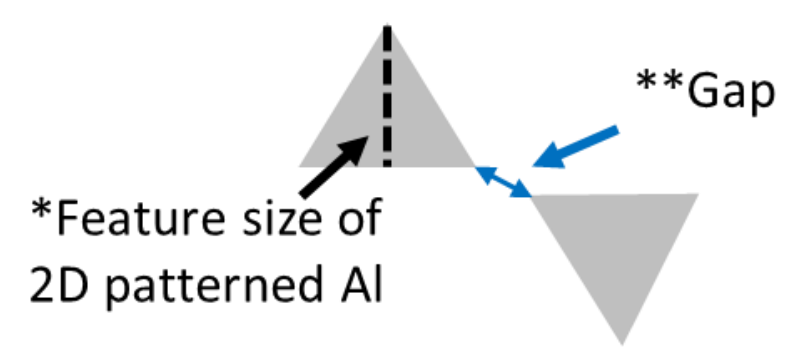




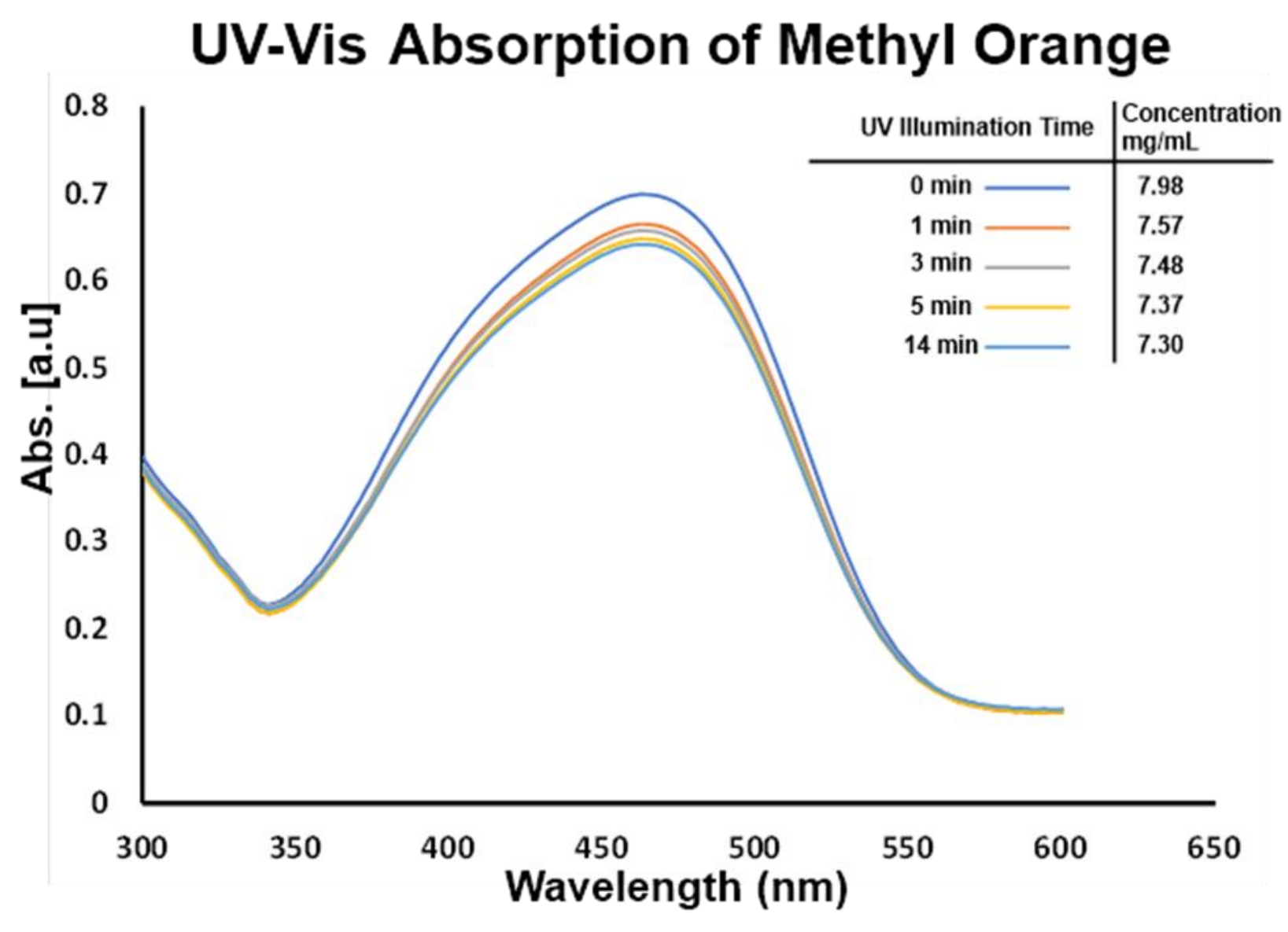

